\title{
The Feasibility Of Telemedicine In Implementation Of Therapeutic Hypothermia For Management Of Neonatal Hypoxic-Ischemic Encephalopathy In Resource-Limited Areas.
}

\section{Adnan Hadid}

King Saud University

Taher S. AL-Shantout

King Saud University

Rayan S. Terkawi

Hamad General Hospital

Baraa M. Aldbes

Maternity and children hospital

Manal M. Zahran ( $D$ montasertoqa1983@gmail.com )

EL-Ekhaa hospital

Fadia A. Alsatouf

EL-Ekhaa hospital

Hani Najjar

Aladan hospital

MHD Hassan Mughrabieh

Syrian Expatriate Medical Association (SEMA)

Nour A. Alhadid

Alfaisal University

Khalid Altirkawi

King Saud University

\section{Research Article}

Keywords: hypoxic-ischemic encephalopathy, therapeutic hypothermia, cooling therapy, NICU,

Telemedicine

Posted Date: February 16th, 2022

DOI: https://doi.org/10.21203/rs.3.rs-1199367/v2 
License: (c) (i) This work is licensed under a Creative Commons Attribution 4.0 International License. Read Full License

Version of Record: A version of this preprint was published at Avicenna Journal of Medicine on February 23rd, 2023. See the published version at https://doi.org/10.1055/s-0042-1760434. 


\section{Abstract}

Background: Telemedicine is widely used in neonatal services in developed countries. Lack of expertise and/or facilities, however, limited its use in developing countries and around areas of military conflicts. To our knowledge, no reports are demonstrating the feasibility of administering therapeutic hypothermia (TH) through telemedicine to neonates with hypoxic-ischemic encephalopathy (HIE) in resource-limited areas.

Methodology: This is a retrospective study, evaluating 22 patients who received $\mathrm{TH}$, guided by telemedicine, through a mobile app $\left(\right.$ Telegram $\left.^{\circledR}\right)$. We assessed the feasibility of utilizing Telemedicine in guiding the application of TH to infants affected with HIE in the North-West of Syria between July 2020 and July 2021.

Results: Out of 5,545 newborn infants delivered during the study period, 22 patients were eligible for TH guided by Telemedicine. Patients were referred for consultation at a median (IQR) of 137 (35-165) minutes of life. A median (IQR) of 12 (3-18) minutes elapsed between the call for a consultation and the consultant response, and a median (IQR) of 30 (0-42) minutes elapsed between seeking the consultation and the initiation of cooling therapy. Eighteen patients completed cooling for 72 hours. The patients' temperatures were within the target range $\left(33-34^{\circ} \mathrm{C}\right)$ most of the time $(84.1 \%)$.

Conclusion: Telemedicine is a feasible method to guide the implementation TH for HIE in resource-limited areas. The short-term success rate is relatively high; however, further studies with a larger population are needed to confirm these findings.

\section{Introduction:}

Hypoxic-ischemic encephalopathy (HIE) is a leading cause of morbidity and mortality worldwide. In 2010, HIE incidence was estimated to be 8.5 cases per 1000 live births; the majority (96\%) were born in low- and middle-income countries.(1) If survived the neonatal period, affected infants are likely to experience an increased burden of neurodevelopmental impairments. Therefore, HIE is not just a disease of the neonatal period but far beyond.

The cornerstone of HIE prevention depends heavily on providing early and adequate antenatal care; the incidence rates of HIE in the United Kingdom have decreased over time from 7.6 per 1000 live births in the 1970 s to 1.9 in the 1990s.(2) However, in areas where antenatal care is not accessible, these rates are still unduly high; thus, efforts to mitigate HIE sequelae are very much needed. Therapeutic Hypothermia (TH), an emerging modality in secondary prevention, is becoming the gold standard of therapy for moderate and severe HIE.(3) Cooling initiated within the first 6 hours of life appears to be effective in reducing the adverse outcomes.(4) To maintain the core temperature within a target, however, cooling must be wellcontrolled in a narrow range $\left(33^{\circ}-34^{\circ} \mathrm{C}\right) .(5)$ The following methods are described: 
Active cooling; using high-technology devices has shown significant improvement in outcomes given the tight control of temperature. $(4,6)$ Unfortunately, cost and infrastructure prevent utilizing them in low- and middle-income countries. Passive cooling, by exposing neonates to room temperature without warmers, was used in several studies and showed a relatively high failure rate.(7) Another technique, referred to as "facilitated cooling", utilizes low-cost cooling devices, such as ice packs, fans, and air conditioners, which showed promising results. For instance, lce packs usage and phase changing materials were described in Brazil and India. $(8,9)$ Tecotherm HELIX cooling device appeared efficient in a trial in India.(10)

Obstacles to the implementation of TH in low-income countries are not limited to the lack of equipment; neonatal intensive care units (NICU) may lack necessary expertise too. Utilizing Telemedicine may fill the gap; using continuous conference video calls was described in level II NICU in underserved areas in the United States, which showed the effectiveness and safety.(11) Telemedicine in HIE management was reported by Craig et al.(12) They used Cisco conferencing video calling software to connect neonatologists with pediatric neurologists. The technologies mentioned, their cost, and the need for infrastructures to operate them, such as high-speed internet, make them inapplicable in certain circumstances, such as conflict areas in northeast Syria, where more creative methods of Telemedicine are needed.

This report describes the difficulties we faced in providing TH in babies with HIE and the solutions we introduced using facilitated cooling techniques with the help of Telemedicine. To our knowledge, this is the first report of its kind from an area of conflict. Its implications may promote the use of TH care and improve these infants' outcomes.

\section{Methods And Settings:}

\section{Study Design, Participants, and Settings}

This is a retrospective, single-center cohort study conducted in El-Ekhaa specialized hospital, a maternity and children hospital in an area ravaged with military conflict in the North-West of Syria, between July 2020 and July 2021. The hospital is located in the middle of dozens of refugee camps, which accommodate about 600,000 refugees. It offers both in-patient and out-patient pediatric and gynecological services. In addition to antenatal care clinics and performing normal deliveries, the hospital services include instrumental and cesarian deliveries and both major and minor gynecological surgeries. The pediatric services include a 25-bed general inpatient service, a 3-bed pediatric intensive care unit (PICU), in addition to the neonatal intensive care unit (NICU) equipped with 10 incubators and conventional and non-invasive mechanical ventilation devices. The NICU offers a wide range of neonatal services, such as phototherapy, exchange transfusion, and surfactant replacement therapy. The hospital also has an emergency department that serves women's and children's emergencies around the clock. It is an academic and research center in the region that previously published on children's and women's health. $(13,14)$ 
Calgary University's HIE calculator was used to facilitate treatment decisions.

(https://play.google.com/store/apps/details?id=com.radsun.hiecalculator\&hl=en_CA\&gl=US)

Patients were cooled if they were $\geq 35$ weeks and a birth weight $\geq 1800$ grams with evidence of perinatal depression as indicated by the presence of either one of the following (criteria A):

1. $\mathrm{PH} \leq 7$ or Base Excess $\leq-16$ on cord blood gas analysis or one performed within 1 hour after delivery; or

2. the need for positive pressure ventilation for $\geq 10$ minutes, or Apgar score $\leq 5$ at 10 minutes

With the presence of (criteria B): Hypoxic ischemic encephalopathy (HIE) stage II or III according to modified Sarnat classification (15), or convulsion.

Patients were excluded if they had severe congenital malformations, high oxygen requirement $\left(\mathrm{FiO}_{2} \geq\right.$ $90 \%$ ), or severe coagulopathy with uncontrolled bleeding.

The treating team consists of in-hospital pediatricians with an online consulting team that includes three neonatologists providing around-the-clock coverage, a radiologist, and other pediatric sub-specialists, including a pediatric neurologist, nephrologist, and hematologist.

If a patient is deemed eligible, the case was discussed with the online on-call neonatologist. Detailed maternal and perinatal histories with all available investigations, including the radiographs, were forwarded through a mobile app (Telegram).

When the decision of cooling was made, the passive cooling process was initiated; the radiant warmer was switched off, followed by transferring the patient to an incubator with starting temperature of $30^{\circ} \mathrm{C}$. Later, the temperature was adjusted as needed to keep the infant's core temperature within the target range $\left(33-34^{\circ} \mathrm{C}\right)$. Morphine was administered as an adjunct therapy to decrease pain and achieve better control of the temperature. When available, a rectal probe was used for continuous monitoring of core temperature; otherwise, a skin probe was used for this purpose, with hourly determinations of core (rectal) temperature using a mercury thermometer. In some cases, if the temperature remained out of range, further measures were taken, including ice bags and adjusting room temperature.

Laboratory investigations performed on all infants included CBC (Complete Blood Count), hepatic and renal function tests, PT (Prothrombin Time), aPTT (activated partial thromboplastin time), and CRP (CReactive Protein). Blood cultures were not performed in any of these infants due to unavailability. However, empiric antibiotics (ampicillin and cefotaxime) were used in all of them due to the tentative diagnosis of "clinical sepsis" and were upgraded in case of clinical deterioration.

Videos of the baby upon admission and later, if he developed convulsion or any abnormal movement, were recorded and forwarded to the neonatologist and pediatric neurologist through the mobile application (Telegram). Plans for treating the convulsions and the duration of the anticonvulsant therapy 
were discussed with the pediatric neurologist. Brain CT (computerized tomography) or MRI (magnetic resonance imaging) scans were also sent to the radiologist for reading through telemedicine.

Age at the consultation requested, time for the neonatologist's response, and the time-lapse between the referral and the point of cooling initiation were recorded.

\section{Outcome measures:}

Short-term outcomes include duration of respiratory support, time to reach full feeding, and survival to discharge. All cases were given an appointment for follow-up in the pediatric clinic.

\section{Statistical Analysis}

We performed descriptive statistics on the total cohort of patients without stratification. Analysis was performed using Excel software (Microsoft Company, Seattle, WA, USA). As the data were non-normally distributed, we reported numerical variables as median and interquartile ranges. Categorical variables were reported as percentages. No meaningful comparisons within the cohort were possible due to the small sample size.

\section{Results:}

During the study period, from July 2020 to July 2021, 5545 pregnant women were delivered in El-Ekhaa hospital; of them, 1290 (23.3\%) were cesarian section deliveries.

Twenty-two patients were referred through Telemedicine for TH eligibility. Records of these patients were analyzed. Patients were referred for consultation at a median (IQR) of 137 (35-165) minutes of life. A median (IQR) of $12(3-18)$ minutes elapsed between the call for a consultation and the consultant response, and a median (IQR) of 30 (0-42) minutes elapsed between seeking the consultation and the initiation of cooling therapy. All the patients who completed TH were followed daily through telemedicine till discharge.

Table 1 presents the patients' characteristics, perinatal management, and cooling process variables. Twenty-two babies were eligible for cooling, 18 (82\%) of them had completed cooling for 72 hours, and 4 $(18 \%)$ babies were excluded. Notably, patients' temperatures remained within the target range $\left(33-34^{\circ} \mathrm{C}\right)$ most of the time ( $84 \%$ of the temperature readings), $5 \%$ of these readings were less than $32^{\circ} \mathrm{C}$, and $11 \%$ of them were above $34^{\circ} \mathrm{C}$ (Figure 1 ). 
Table 1

Patients' characteristics, perinatal management, and cooling process

\begin{tabular}{|ll|}
\hline N & 18 \\
\hline gestational age, weeks; median (IQR) & $38(38-40)$ \\
\hline birthweight, grams; median (IQR) & $3,400(3,200-3,500)$ \\
\hline Male, N (\%) & $13(72)$ \\
\hline Primigravida; N (\%) & $8(44)$ \\
\hline Vaginal delivery; N (\%) & $17(94)$ \\
\hline Meconium-stained amniotic fluid; N (\%) & $7(39)$ \\
\hline Resuscitation initiated by a doctor; N (\%) & $3(17)$ \\
\hline Apgar's score at 1; median (IQR) & $3(3-3.5)$ \\
\hline Apgar's score at 5; median (IQR) & $5(4-5.25)$ \\
\hline Apgar's score at 10; median (IQR) & $6(5-6)$ \\
\hline Positive pressure ventilation (PPV) > 10 min; N (\%) & $15(83)$ \\
\hline Chest Compressions; N (\%) & $1(6)$ \\
\hline Cord blood gases; N (\%) & $5(28)$ \\
\hline Arterial blood gases in 1h; N (\%) & $11(61)$ \\
\hline pH; median (IQR) & $7.09(7.07-7.18)$ \\
\hline Base deficit; median (IQR) & $21(17-25)$ \\
\hline Serum HCO ${ }_{3} ;$ median (IQR) & $14(78)$ \\
\hline Age at cooling, hours; median (IQR) & $1(17-7-9.6)$ \\
\hline Time to reach Cooling range, hours; median (IQR) & $1(1-3)$ \\
\hline The rectal sensor used (to regulate temperature); N (\%) & $10(56)$ \\
\hline Progressed to stage 3 of encephalopathy; N (\%) & $1(6)$ \\
\hline Had convulsions, N (\%) & $15(83)$ \\
\hline Had convulsions before 6h of age; N (\%) & $3(17)$ \\
\hline Anti-seizure drugs used, \% & 14 (I) \\
\hline N: Number, IQR: Interquartile, HCO3: Bicarbonate. & \\
\hline
\end{tabular}


Most of the deliveries were vaginal; only one patient (6\%) was delivered by C-section. Fifteen out of the 18 (83\%) patients required positive pressure ventilation (PPV) for more than 10 minutes, and one patient $(6 \%)$ required chest compression. When needed, resuscitation was initiated by the treating physician only in $3(17 \%)$ cases and the rest by delivering midwife or nurse. Convulsions have been observed in 15 patients (83\%); 14 of them were started on an anticonvulsant medication.

Table 2 summarizes management at the NICU and the outcomes. Out of the 18 patients admitted to NICU, 15 (83\%) required respiratory support, and 17 (94\%) were sedated by morphine during the cooling procedure. Furthermore, 11 (61\%) patients received fresh frozen plasma due to coagulation derangement. Seventeen (94\%) patients survived to discharge, and all of them were followed in the clinic.

Table 2

Nursey management and outcomes

\begin{tabular}{|ll|}
\hline Respiratory support, N (\%) & $15(83)$ \\
\hline$\cdot$ Mechanical ventilation; N (\%) & $10(56)$ \\
\hline$\cdot$ Continuous positive airway pressure (CPAP); N (\%) & $1(6)$ \\
\hline • Nasal cannula; N (\%) & $4(22)$ \\
\hline Mechanical Ventilation days & $4(3-6)$ \\
\hline Sedation/analgesia; N (\%) & $17(94)$ \\
\hline Vasopressors and Inotropes; N (\%) & $2(11)$ \\
\hline Extended-spectrum antibiotics; N (\%) & $10(56)$ \\
\hline Duration of antibiotics therapy, days; median (IQR) & $10(7-15)$ \\
\hline Fresh Frozen Plasma; N (\%) & $11(61)$ \\
\hline Age at feeds initiation, days; median (IQR) & $5(4-6)$ \\
\hline Age when target feeds reached, days; median (IQR) & $7(6-10)$ \\
\hline Feeding at discharge: & \\
\hline - Breast milk; N (\%) & $13(71)$ \\
\hline • Mixed; N (\%) & $2(12)$ \\
\hline • Formula; N (\%) & $2(12)$ \\
\hline Length of hospital stay, days; median (IQR) & $12(9-17)$ \\
\hline Survived; N (\%) & $17(94)$ \\
\hline Follow up; N (\%) & 100 \\
\hline N: Number, IQR: Interquartile. & \\
\hline
\end{tabular}


Table 3 presents the most important laboratory findings and imaging studies. All survived patients had brain imaging, 4 (22\%) had a sonogram, $6(33 \%)$ had a CT-scan, and $11(61 \%)$ had an MRI.

Table 3

Laboratory and imaging

\section{Highest WBCs, $\times 10^{6} / \mathrm{L}$; median (IQR)}

Lowest WBCs, $\times 10^{6} / \mathrm{L}$; median (IQR)

Lowest platelets, $\times 10^{6} / \mathrm{L}$; median (IQR)

PT, seconds; median (IQR)

aPTT, seconds; median (IQR)

INR; median (IQR)

Admission CRP, mg/L, median (IQR)

Highest CRP, mg/L, median (IQR)

Admission creatinine, $\mathrm{mg} / \mathrm{dl}$, median (IQR)

Highest creatinine, mg/dl, median (IQR)

Admission AST, U/L, median (IQR)

Highest AST, U/L, median (IQR)

Admission ALT, U/L, median (IQR)

Highest ALT, U/L, median (IQR)

Head sonogram, \%

Brain CT, \%

Brain MRI, \%

Age at brain imaging, days; median (IQR)

WBC: White blood cells, PT: Prothrombin time, aPTT: Partial thromboplastin time, INR: international normalized ratio, CRP: C-reactive protein, AST: Aspartate aminotransferase, ALT: Alanine Aminotransferase, CT: computed tomography, MRI: Magnetic resonance imaging.
$14.8(12.8-19.5)$

$7.3(6-9.1)$

159 (105-176)

$25(20-34)$

$36.5(36-42$.

$2.1(1.6-2.3)$

$2.3(1.1-10.3)$

$21.5(9.3-42.8)$

$0.8(0.7-0.9)$

$0.9(0.7-1)$

$80(47-102)$

$80(47-102)$

$40(21-57)$

$43(21-68)$

$4(22)$

$6(33)$

$11(61)$

$9.5(7-14)$

\section{Discussion:}

The significant gaps in maternal and neonatal health care are prominent in northwest Syria. However, few qualitative reports described the catastrophe. Direct threats to medical care professionals and killing many of them led to the displacement of others. These shortages in providers added to the destruction of many facilities and lack of essential equipment, left the whole healthcare system with major deficiencies, $(16,17)$ especially in terms of delivering tertiary care for the most vulnerable populations, such as infants 
with HIE.(18) Providing quality medical education to the providers who decided to stay and handle the burden was a challenge that was difficult, to say the least. Therefore, we utilized Telemedicine tools hoping to fill some of the critical gaps. This report demonstrates a successful application of TH through Telemedicine.

Worldwide, Telemedicine has been used for some neonatal services, such as the screening for retinopathy of prematurity (ROP), Tele-echocardiography, newborn resuscitation, and even for family support.(19) Tele-rounding on NICU patients was an effective and safe way of care as well.(11) An undergoing clinical trial to explore the feasibility and efficacy of Telemedicine in complicated situations in NICU is registered on (ClinicalTrials.gov).(20) In our situation, the unavailability of a neonatologist on-site, and the logistic difficulties encountered upon transferring critically ill infants to the nearest tertiary center across the borders to Turkey, required an innovative approach to these challenging conditions. Also, a valid application of therapeutic hypothermia requires it to be applied within 6 hours of birth, meaning that transferring the infant across the border is impractical. Thus, the option to initiate TH through Telemedicine guidance became the only option in hand. This was quite challenging for both the treating team in the field and the neonatology consultants abroad; they had to liaise together from the time of patient's admission till discharge. However, despite the insufficient experience and the paucity of reports to inform the management of such critical cases in the NICU, we were able to pull it together and initiate the project.

Unlike in developed countries, where high-tech equipment for Telemedicine is readily available, we had to settle for a less complicated system and improvise special ways of effective consultation. We provided the treating staff with online lectures about HIE, the treatment protocol, and how to evaluate the patient's eligibility for TH. Discussing the case with the neonatologist and subspecialty consultants through the mobile App (Telegram) and using the HIE calculator to facilitate decisions have smoothened the course of therapy; the plan of care was adjusted in a timely fashion based on the clinical progress and the results of the investigations. Furthermore, applying this therapy through Telemedicine helped us reduce the number of cases to be transferred across the border, with the inherent associated risks of this process. More importantly, it enhanced the bonding with families and increased parents' satisfaction as well.

On average, the time lapse between seeking consultants' guidance and the initiation of cooling was relatively short, about 30 minutes. In most instances, it took the consultant to respond to the help call only 12 minutes, an impressive time frame in such a setting.

The incidence of HIE in this population is obviously higher than that in developed countries. The lack of effective therapies to mitigate the consequences of HIE provide a great impetus to create and maintain such a project; its implementation, though challenging, was practically within reach. For example, the majority of patients remained within the targeted temperature range for $84 \%$ of the cooling duration. This rate is comparable to what is reported by studies in low-income settings. A study from Brazil using lowcost materials for cooling (ice packs) reported staying in the targeted range in $82.3 \%$ of the time.(8) 
Similarly, in a study from India, ice packs cooling maintained temperature within the range in $92.5 \%$ of measurements.(9)

Implementing TH to reduce mortality and/or morbidity was emphasized in a couple of large clinical trials in high-income countries. $(4,6,21)$ A Cochrane review in 2013 pooled data from 11 pilot studies, mostly from high-income countries, and concluded that findings are in favor of using TH.(22) A meta-analysis was published in the same year has pooled data for 7 studies from low-income countries (23) and concluded that further studies are required in similar settings. Recently, a large meta-analysis showed overall benefits from TH after pooling 28 studies in different countries.(24) In that study, subgroups analysis revealed possible benefits from TH in low and middle-income countries. Surprisingly, a large clinical trial was conducted in India, Sri Lanka, and Bangladesh recommended against TH in low and middle-income countries due to increased mortality and morbidity.(25) Of note, almost half of infants in each arm ended up with death or moderate to severe neurodevelopmental compromise, and the death rate in the TH group was $42 \%$ versus $31 \%$ in the control group.

Apparently, there is considerable heterogeneity in results from low and middle-income countries, specifically in mortality rates. While the TH procedure is well-defined and almost unified among studies in high-income studies, various techniques were used in middle and low-income ones. However, linking outcomes to the economic status of the origin country may not sound realistic. Furthermore, mortality in patients with HIE can be multifactorial, and care provided is less standardized among those countries.

In our study, we were unable to produce reliable conclusions in terms of mortality and long-term outcomes beyond the neonatal period, mainly due to a lack of expertise in pediatric development objective assessment, an area where additional efforts are needed. Other limitations of this study include a small sample size and the lack of a control group. Larger studies with robust design are needed to corroborate our findings and to shed more light on the need of this unprivileged population.

\section{Conclusions:}

In limited resources or conflict areas, applying therapeutic hypothermia to manage HIE is feasible through Telemedicine means utilizing simple technologies, such as mobile Apps. Committed teams of experts leasing with the treating team in the NICU is a prerequisite for success. This practice seems a promising modality that has the potential to save lives and improve the quality of care in war-ravaged zones.

\section{List Of Abbreviations:}

TH: therapeutic hypothermia

HIE: hypoxic-ischemic encephalopathy

IQR: Interquartile 
${ }^{\circ} \mathrm{C}$ : Degree Celsius

NICU: neonatal intensive care units

PICU: pediatric intensive care unit

$\mathrm{FiO}_{2}$ : fraction of inspired oxygen

PT: Prothrombin Time

aPTT: activated partial thromboplastin time

CRP: C-Reactive Protein

CT: computerized tomography

MRI: magnetic resonance imaging

SEMA: Syrian expatriate medical association

IRB: Institutional Review Board

PPV: positive pressure ventilation

ROP: retinopathy of prematurity

$\mathrm{N}:$ Number

HCO3: Bicarbonate

WBC: White blood cells

INR: international normalized ratio

AST: Aspartate aminotransferase

ALT: Alanine Aminotransferase

\section{Declarations:}

- Ethics approval and consent to participate: The Institutional Review Board (IRB) of the Syrian expatriate medical association (SEMA) has approved the study with an exemption from obtaining anything other than general consent for treatment upon hospitalization. The IRB study number:

(2020-02). Data were collected from the patients' files and was reviewed by two doctors. All methods were performed in accordance with the Declaration of Helsinki.

- Consent for publication: Not applicable. 
- Availability of data and materials: As per the local institutional regulations; data are not publicly available, but to be provided upon request from the corresponding author for research purposes in deidentified form.

- Competing interests: Authors have no competing interest to disclose.

- Funding: This study received no fund.

- Authors' contributions: Data collection was done by M. Z. and F. A. Data processing and analysis were done by K. A. and A. H. Figure 1 was prepared by A. H. All authors participated in the discussions and drew the conclusions as well as manuscript the article and agreed on the contents.

- Acknowledgements: We thank the staff of EL-Ekhaa hospital for their support.

- Authors' information (optional): Not applicable.

\section{References:}

1. Lee ACC, Kozuki N, Blencowe H, Vos T, Bahalim A, Darmstadt GL, et al. Intrapartum-related neonatal encephalopathy incidence and impairment at regional and global levels for 2010 with trends from 1990. Pediatric research. 2013 Dec;74 Suppl 1(Suppl 1):50-72.

2. Kurinczuk JJ, White-Koning M, Badawi N. Epidemiology of neonatal encephalopathy and hypoxicischaemic encephalopathy. Early human development. 2010 Jun;86(6):329-38.

3. Wassink G, Davidson JO, Dhillon SK, Zhou K, Bennet L, Thoresen M, et al. Therapeutic Hypothermia in Neonatal Hypoxic-Ischemic Encephalopathy. Current Neurology and Neuroscience Reports. 2019 Jan 14;19(1):2.

4. Azzopardi D V, Strohm B, Edwards AD, Dyet L, Halliday HL, Juszczak E, et al. Moderate hypothermia to treat perinatal asphyxial encephalopathy. The New England journal of medicine. 2009 Oct 1;361(14):1349-58.

5. Shankaran S, Laptook AR, Pappas A, McDonald SA, Das A, Tyson JE, et al. Effect of Depth and Duration of Cooling on Death or Disability at Age 18 Months Among Neonates With HypoxicIschemic Encephalopathy: A Randomized Clinical Trial. JAMA. 2017 Jul 4;318(1):57-67.

6. Shankaran S, Laptook AR, Ehrenkranz RA, Tyson JE, McDonald SA, Donovan EF, et al. Whole-body hypothermia for neonates with hypoxic-ischemic encephalopathy. The New England journal of medicine. 2005 Oct 13;353(15):1574-84.

7. Lumba R, Mally P, Espiritu M, Wachtel E V. Therapeutic hypothermia during neonatal transport at Regional Perinatal Centers: active vs. passive cooling. Journal of perinatal medicine. 2019 Apr 24;47(3):365-9.

8. Kinoshita AN, Magalhães M, Rodrigues DP, Variane GFT, Pietrobom RFR, Gallaci CB, et al. Effectiveness of reaching and maintaining therapeutic hypothermia target temperature using lowcost devices in newborns with hypoxic-ischemic encephalopathy. Anatomical record (Hoboken, NJ: 2007). 2021;304(6):1217-23. 
9. Prashantha YN, Suman Rao PN, Nesargi S, Chandrakala BS, Balla KC, Shashidhar A. Therapeutic hypothermia for moderate and severe hypoxic ischaemic encephalopathy in newborns using lowcost devices - ice packs and phase changing material. Paediatrics and international child health. 2019 Oct 2;39(4):234-9.

10. Oliveira V, Kumutha JR, E N, Somanna J, Benkappa N, Bandya P, et al. Hypothermia for encephalopathy in low-income and middle-income countries: feasibility of whole-body cooling using a low-cost servo-controlled device. BMJ paediatrics open. 2018;2(1):e000245.

11. Makkar A, McCoy M, Hallford G, Escobedo M, Szyld E. A Hybrid Form of Telemedicine: A Unique Way to Extend Intensive Care Service to Neonates in Medically Underserved Areas. Telemedicine journal and e-health: the official journal of the American Telemedicine Association. 2018 Sep 1;24(9):71721.

12. Craig AK, McAllister LM, Evans S, Melendi ME. Telemedicine consults to assess neonatal encephalopathy are feasible in the neonatal intensive care unit. Journal of perinatology: official journal of the California Perinatal Association [Internet]. 2021 Jun 1 [cited 2021 Jul 20];41(6):151921. Available from: https://pubmed.ncbi.nlm.nih.gov/32958837/

13. Terkawi AS, Bakri B, Alsadek AS, Al-Hasan AH, Alrahhal MS, Alsaleh FM, et al. Child and adolescent health in northwestern Syria: Findings from Healthy-Syria 2017 study. Avicenna Journal of Medicine. 2019 Apr 9;9(02):61-74.

14. Terkawi AS, Bakri B, Alsadek AS, Alsibaee RH, Alasfar EM, Albakour AH, et al. Women's health in Northwestern Syria: Findings from Healthy-Syria 2017 study. Avicenna Journal of Medicine. 2019 Jul 9;9(03):94-106.

15. Sarnat HB, Sarnat MS. Neonatal encephalopathy following fetal distress. A clinical and electroencephalographic study. Archives of neurology. 1976 Oct;33(10):696-705.

16. Medical Personnel Are Targeted in Syria [Internet]. Physicians for Human Rights. 2021. Available from: https://phr.org/our-work/resources/medical-personnel-are-targeted-in-syria/

17. FM F, A S, A T, M A, F E-J, AP C, et al. Health workers and the weaponisation of health care in Syria: a preliminary inquiry for The Lancet-American University of Beirut Commission on Syria. Lancet (London, England). 2017 Dec 2;390(10111):2516-26.

18. Y $B, D R, A S, L M, F F, W Z$, et al. Challenges of providing healthcare worker education and training in protracted conflict: a focus on non-government controlled areas in north west Syria. Conflict and health [Internet]. 2020 Jul 8 [cited 2021 Oct 8];14(1). Available from: https://pubmed.ncbi.nlm.nih.gov/32665784/

19. Makkar A, Siatkowski RM, Szyld E, Ganguly A, Sekar K. Scope of telemedicine in neonatology. Zhongguo dang dai er ke za zhi = Chinese journal of contemporary pediatrics. 2020 May;22(5):396408.

20. Craig A, Ngo T. Telemedicine to Improve Use of Therapeutic Hypothermia in Rural Settings [Internet]. ClinicalTrials.gov identifier (NCT number): NCT03706417. 2018 [cited 2021 Nov 7]. Available from: https://clinicaltrials.gov/ct2/show/study/NCT03706417 
21. PD G, JS W, D A, R B, AD E, DM F, et al. Selective head cooling with mild systemic hypothermia after neonatal encephalopathy: multicentre randomised trial. Lancet (London, England). 2005 Feb 19;365(9460):663-70.

22. SE J, M B, R H, WO T-M, TE I, PG D. Cooling for newborns with hypoxic ischaemic encephalopathy. The Cochrane database of systematic reviews. 2013 Jan 31;2013(1).

23. SS P, S S, A W, EB C, S T. Therapeutic hypothermia for neonatal encephalopathy in low- and middleincome countries: a systematic review and meta-analysis. PloS one. 2013 Mar 19;8(3).

24. BB A, M B, B G, A MK, M K, T G, et al. Effects of therapeutic hypothermia on death among asphyxiated neonates with hypoxic-ischemic encephalopathy: A systematic review and metaanalysis of randomized control trials. PloS one. $2021 \mathrm{Feb}$ 1;16(2).

25. Thayyil S, Pant S, Montaldo P, Shukla D, Oliveira V, Ivain P, et al. Hypothermia for moderate or severe neonatal encephalopathy in low-income and middle-income countries (HELIX): a randomised controlled trial in India, Sri Lanka, and Bangladesh. The Lancet Global health. 2021;9(9):e1273-85.

\section{Figures}

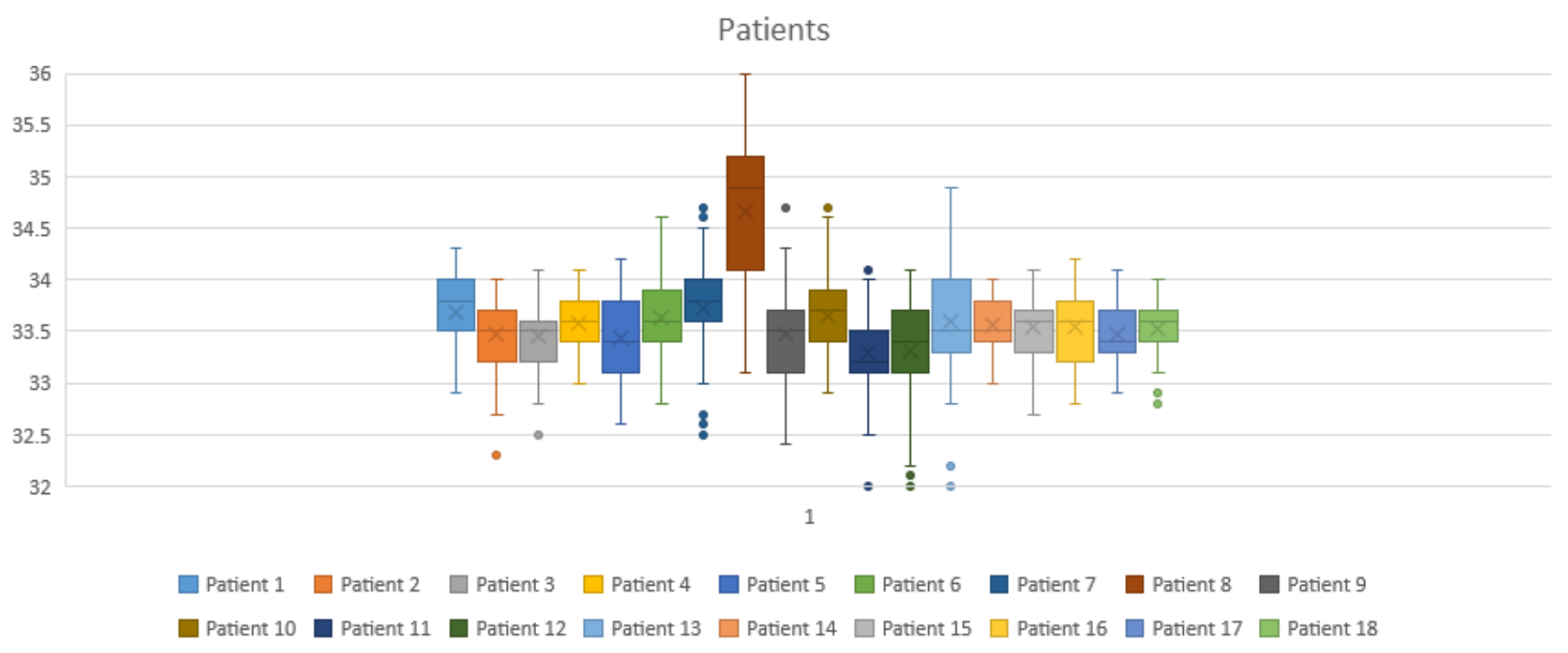

Figure 1

Temperature readings for every patient during Therapeutic Hypothermia. 